\title{
Optimal scheduling method based on building virtual energy storage equivalent battery
}

\author{
Wei He ${ }^{1}$, Jia $\mathrm{Li}^{1}$, Weizhe Zhao ${ }^{1}$ and Yaqing Zhang ${ }^{2, *}$ \\ ${ }^{1}$ State Grid Jiangxi Electric Power Research Institute, Jiangxi, China \\ ${ }^{2}$ Key Laboratory of Smart Grid of Ministry of Education, Tianjin University, Tianjin, China
}

\begin{abstract}
In this paper, an optimal scheduling method based on building virtual energy storage equivalent battery is proposed. Firstly, the thermal load prediction model is built based on the thermodynamic model, and then the equivalent battery model of virtual energy storage is established by combining with inverter air conditioning system model. Then, with the goal of minimizing the user's electricity cost, considering the constraints of energy storage state and charging and discharging power of equivalent battery model, an optimal scheduling method of building virtual energy storage equivalent battery is constructed. The results show that the proposed method can predict the building thermal load, update the building virtual energy storage equivalent battery parameters, formulate the corresponding optimal scheduling strategy, and reduce the electricity cost of users for heating on the premise of ensuring the thermal comfort of users.
\end{abstract}

\section{Introduction}

Energy is the basis for human survival and the lifeblood of the national economy [1]. With economic development and progress, people's requirements for quality of life continue to increase, and building energy consumption is increasing year by year. Building energy has become one of the world's largest energy consumption fields, accounting for more than one-third of global terminal energy consumption [2]. This problem also exists in China. According to the statistics, China's building construction and operation energy accounted for $37 \%$ of the total energy consumption of the whole society in 2018, and operating energy consumption accounted for $23 \%$. Among them, the heating energy consumption of northern cities and towns accounts for about $21 \%$ of the total energy consumption of buildings in the country [3]. In northern China, coal-fired heating is usually used for heating in cities and towns, which makes building energy consumption rise and environmental pollution is becoming increasingly serious. In response to this problem, China proposed a solution to "replace coal with electricity and clean heating" [4], using non-polluting, low-carbon emissions and strong controllable electric heating instead of traditional coal-fired heating to solve coal burning Environmental pollution caused by heating.

However, with the use of electric heating, the electricity load of the building increases, and the user's electricity cost also increases [5]. At the same time, due to the thermal inertia of the building, it can provide some heat storage capacity. By making full use of the heat storage capacity provided by building, the thermal load in peak period can be transferred to normal period or low period. On this basis, combined with the electricity price policy, it can reduce the user's electricity cost while meeting the thermal comfort needs of the building [6].

At present, many scholars have studied the methods of using the heat storage potential of buildings to reduce heating cost. Literature [7] proposed the concept of energy controller for optimal scheduling and verifies that the use of buildings for heat storage is an economical and rapid way to save energy and reduce heating cost. Literature [8] studies the upper and lower limits of comfort that can be sacrificed in general buildings and formulates control strategies on this basis. The result shows that some strategies can achieve building energy saving while ensuring the comfort of the building environment. Literature [9] studies the almost zero energy consumption single-family houses equipped with photovoltaic cells and found that the heat storage of building has great potential, which can realize the consumption of renewable energy and peak-shaving and valley-filling during peak heating periods.

based on the above research, some scholars have proposed the use of virtual energy storage ideas to quantify the building heat storage potential. Literature [10] built a virtual energy storage model considering the building envelope, and proposed to use charge and discharge power to represent the virtual energy storage capacity of building at each time. Considering the building age and temperature setting scheme, literature [11] proposed to quantify the virtual energy storage capacity of building as available storage capacity, storage efficiency and power transfer capacity.

However, the quantitative method of building virtual energy storage capacity proposed in the existing research

\footnotetext{
* Corresponding author: zhangyq111@tju.edu.cn
} 
is not unified and compatible with the original optimal scheduling model. This brings additional work.

In this paper, building virtual energy storage equivalent battery is constructed with reference to the battery model, and the optimal scheduling method considering building virtual energy storage equivalent battery is proposed. Firstly, building thermal load forecasting model is constructed based on thermodynamic model; secondly, building virtual energy storage equivalent battery model is constructed by analogy with battery model; and then the optimal scheduling method of building virtual energy storage equivalent battery is proposed with the objective of minimizing the user's electricity cost. Results and discussion shows that the proposed method is compatible with the original optimal scheduling model, which can quantify the building heat storage potential, fully explore the building virtual energy storage capacity, and reduce the user's electricity cost for heating.

\section{Methods}

Battery is one of the most commonly energy storage equipment. The original optimal scheduling model of building often includes such equipment. Therefore, by analogy with the battery model, the thermal inertia of the building envelope is quantified as the building virtual energy storage equivalent battery model to participate in the optimal scheduling of building. Under the premise of ensuring the thermal comfort of users, the virtual energy storage capacity of building can be fully explored, and the original optimal scheduling model of the building can be compatible. Fig 1 shows the framework of optimal scheduling method based on building virtual energy storage equivalent battery model.

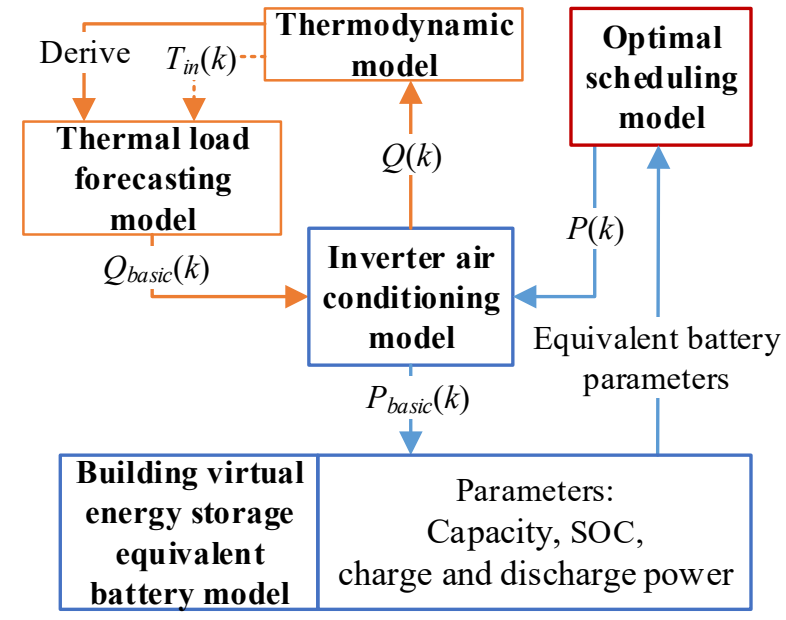

Fig. 1. Framework of optimal scheduling method based on building virtual energy storage equivalent battery

\subsection{Thermal load forecasting model}

By analyzing the heat exchange process of building in winter heating period, the simplified equivalent thermodynamic model[12] of building can be expressed by (1).

$$
C \frac{d T_{\text {in }}}{d t}=Q-\frac{\left(T_{\text {in }}-T_{\text {out }}\right)}{R}
$$

Where, Where, $C$ is the building equivalent heat capacity, $\mathrm{kJ} /{ }^{\circ} \mathrm{C} ; T_{\text {in }}$ is the indoor temperature, ${ }^{\circ} \mathrm{C} ; T_{\text {out }}$ is the outdoor temperature, ${ }^{\circ} \mathrm{C} ; R$ is the building equivalent thermal resistance, ${ }^{\circ} \mathrm{C} / \mathrm{kW} ; Q$ is the heating power, $\mathrm{kW}$.

According to (1) and heat dissipation principle of building envelope, the thermal load of period $k$ can be expressed by (2).

$$
Q_{\text {thermal }}(k)=\alpha F K\left(T_{\text {in. } i}(k)-T_{\text {out }}(k)\right) / 1000
$$

Where, $Q_{\text {thermal }}(k)$ is the thermal load of period $k$, kW; $\alpha$ is the correction coefficient of housing temperature difference; $K$ is the heat transfer coefficient of the house, $\mathrm{W} /\left(\mathrm{m}^{2} \times{ }^{\circ} \mathrm{C}\right) ; F$ is the area of the building envelope structure, $\mathrm{m}^{2} ; T_{\text {in }}(k)$ is the indoor temperature of period $k,{ }^{\circ} \mathrm{C} ; T_{\text {out }}(k)$ is the outdoor temperature of period $k,{ }^{\circ} \mathrm{C}$. It is assumed that the outdoor temperature of period $k$ is a constant value.

\subsection{Inverter air conditioning model}

The main electric power equipment of the inverter air conditioning system is the compressor. In order to simplify the calculation process, the electric power of the remaining equipment of the inverter air conditioning system is ignored, and the relationship between the electric power and the operating frequency of the compressor is simplified to a linear relationship[12], as shown in equation (3).

$$
P=a_{1} f+b_{1}
$$

Where, $P$ is the electric power of the inverter air conditioning system, $\mathrm{kW} ; a_{1}$ and $b_{1}$ are constant coefficients.

Similar to the electric power, the relationship between the heating power of the inverter air conditioning system and the operating frequency of the compressor can also be simplified as a linear relationship, as shown in equation (4).

$$
Q_{\text {iav }}=a_{2} f+b_{2}
$$

Where, $Q_{i a v}$ is the heating power of the inverter air conditioning system, $\mathrm{kW} ; a_{2}$ and $b_{2}$ are constant coefficients.

Combining equations (3) and (4), the relationship between the electric power and the heating power of the inverter air conditioning system can be expressed by equation (5).

$$
Q_{i a v}=\frac{a_{2}}{a_{1}} P-\frac{a_{2} b_{1}}{a_{1}}+b_{2}
$$

\subsection{Building virtual energy storage equivalent battery model}

\subsubsection{Capacity}

The range of indoor comfort is $\left[T_{\min }, T_{\max }\right]$, and it is assumed that $T_{i n}(k)$ is the initial value of room temperature of period $k$. When $T_{i n}(k)$ and $T_{\min }$ are equal, the equivalent battery storage capacity is zero; when $T_{i n}(k)$ and $T_{\max }$ are equal, the equivalent battery storage 
capacity is the largest, which is the capacity of equivalent battery, as shown in (6).

$$
C_{c a}=C\left(T_{\max }-T_{\min }\right)
$$

Where, $C_{c a}$ is the capacity of equivalent battery, kJ.

\subsubsection{SOC}

SOC describes the state of charge (SOC) of equivalent battery, which is defined as the ratio of the storage capacity of equivalent battery to the capacity of equivalent battery. It can be obtained by (7).

$$
\operatorname{SOC}(k)=\frac{E(k)}{C_{c a}}
$$

Where, $E(k)$ is the initial storage capacity of equivalent battery of period $k, \mathrm{~kJ}$.

Combined with (1) and the definition of capacity, the initial storage capacity of equivalent battery of period $k$ can be expressed by (8).

$$
E(k)=C\left(T_{\text {in }}(k)-T_{\text {min }}\right)
$$

\subsubsection{Charge and discharge power}

The heating load that keeps the actual room temperature the same as the user's set room temperature is defined as the reference heating power. According to (2), reference heating power of period $k$ can be expressed by (9).

$$
Q_{\text {basic }}(k)=\alpha F K\left(T_{\text {set }}-T_{\text {out }}(k)\right) / 1000
$$

Where, $Q_{\text {basic }}(k)$ is the reference heating power of period $k, \mathrm{~kW}$.

Combined with (5) and (9), the reference electric power of period $k$ can be expressed by (10).

$$
P_{\text {basic }}(k)=\frac{a_{1} \alpha F K\left(T_{\text {set }}-T_{\text {out }}(k)\right) / 1000}{a_{2}}-\frac{a_{1} b_{2}}{a_{2}}+b_{1}
$$

When electric power deviates from the reference electric power, the building equivalent battery is in the state of charging or discharging. When the electric power is greater than the reference electric power, the equivalent battery is in the state of charging, otherwise it is in the state of discharging. The equivalent charge and discharge power can be expressed by (11).

$$
P_{c / d i s}(k)=P(k)-P_{\text {basic }}(k)
$$

Where, $P(k)$ is the actual electric power of inverter air conditioning of period $k, \mathrm{~kW} ; P_{c / d i s}(k)$ is the equivalent charge and discharge power, $\mathrm{kW}$.

\subsubsection{Update of SOC}

By using the parameter of SOC, the basic condition of equivalent battery can be quickly transferred between two adjacent periods ( $\mathrm{k}$ and $\mathrm{k}+1)$. According to (7), SOC of building equivalent battery can be updated by (12).

$S O C(k+1)=\frac{E(k+1)}{C_{c a}}=\frac{E(k)+\Delta E(k)}{C_{c a}}=S O C(k)+\frac{\Delta E(k)}{C_{c a}}(12)$

Where, $\Delta E(k)$ is the increase of storage capacity of period $k, \mathrm{~kJ}$.
$\Delta E(k)$ is composed of building self-discharge loss (i.e. building heat dissipation) and heating capacity of inverter air conditioning, as shown in (13).

$$
\Delta E(k)=\int_{k \Delta t}^{(k+1) \Delta t}\left(Q(t)-Q_{\text {diss }}(t)\right) d t
$$

Where, $Q(t)$ is the heating power of inverter air conditioning at time $t, \mathrm{~kW} ; Q_{\text {diss }}(t)$ is the self discharge power (heat dissipation speed) of building at time $t, \mathrm{~kW}$.

Assuming that the heating power is constant in period $k$, according to equations (5) and (10), the heating power at time $t$ can be expressed by (14).

$$
Q(t)=Q(k)=\frac{a_{2}}{a_{1}}\left(P_{c / d i s}(k)+P_{\text {basic }}(k)\right)-\frac{a_{2} b_{1}}{a_{1}}+b_{2}
$$

The self discharge power of building at time $t$ can be expressed by (15).

$$
Q_{\text {diss }}(t)=\alpha F K\left(T_{\text {in }}(t)-T_{\text {out }}(t)\right) / 1000
$$

Where, $T_{\text {out }}(t)$ is the outdoor temperature at time $t,{ }^{\circ} \mathrm{C} ; T_{\text {in }}(t)$ is the indoor temperature at time $t,{ }^{\circ} \mathrm{C}$.

Assuming that the outdoor temperature is constant during the period $k,(16)$ can be obtained.

$$
T_{\text {out }}(t)=T_{\text {out }}(k) \quad(k \Delta t \leq t \leq(k+1) \Delta t)
$$

Solve the simplified equivalent thermodynamic model shown in (1), $T_{i n}(t)$ in (15) can be calculated by equation (17).

$$
T_{\text {in }}(t)=\left(T_{\text {in }}(0)-T_{\text {out }}(k)-R Q(t)\right) e^{-\frac{t}{R C}}+T_{\text {out }}(k)+R Q(t)
$$

Where, $T_{i n}(0)$ is the initial indoor temperature of period $k$, which can be obtained by $(18),{ }^{\circ} \mathrm{C}$.

$$
T_{\text {in }}(0)=T_{\text {in }}(k)=T_{\text {min }}+S O C(k)\left(T_{\text {max }}-T_{\text {min }}\right)
$$

\subsection{Optimal scheduling model}

\subsubsection{Objective function}

The objective function of optimal scheduling is that the user's heating cost is the smallest, and the objective function can be expressed by (19).

$$
\min \text { cost }=\sum P(k) \times \Delta t \times c(k)
$$

Where, $c(k)$ is the electricity price of period $k$, yuan/kWh.

\subsubsection{Constraints}

1) SOC constraint

When there are personnel activities inside the building, the indoor temperature should be ensured within the indoor comfortable temperature range. According to the definition of SOC above, the indoor comfortable temperature range constraint is included in the SOC constraint, as shown in (20).

$$
0 \leq \operatorname{SOC}(k) \leq 1
$$

2) Charge and discharge power limit constraints

The charging and discharging power is affected by the adjustable frequency range of the inverter air conditioning system and the maximum output of the photovoltaic at each time, and it needs to satisfy equations (21)-(23).

$$
-P_{\text {dis } \max }(k) \leq P_{c / d i s}(k) \leq P_{c \text { max }}(k)
$$




$$
\begin{aligned}
& P_{\text {dis } \max }(k)=P_{\text {basic }}(k)-P_{\text {min }} \\
& P_{c \text { max }}(k)=P_{\text {max }}-P_{\text {basic }}(k)
\end{aligned}
$$

Where, $P_{d i s \max }(k)$ is the maximum discharge power, $\mathrm{kW} ; P_{c \max }(k)$ is the maximum charging power, $\mathrm{kW}$; $P_{\min }$ is the minimum input power of the inverter air conditioning system, $\mathrm{kW} ; P_{\max }$ is the maximum input power of the inverter air conditioning system, $\mathrm{kW}$.

\section{Results and discussion}

\subsection{Case study}

The six stories single office building in literature [13] is selected as the research object. The office building is $49.5 \mathrm{~m}$ long and $10.8 \mathrm{~m}$ wide. Each floor of the office building is $3.6 \mathrm{~m}$ high. The values of parameters in model is shown in Table 1.

Table 1. The values of parameters in model

\begin{tabular}{|c|c|c|c|c|c|c|}
\hline $\begin{array}{c}\text { Paramet } \\
\text { er }\end{array}$ & $C /\left(\mathrm{kJ} /{ }^{\circ} \mathrm{C}\right)$ & $R /\left({ }^{\circ} \mathrm{C} / \mathrm{kW}\right)$ & $a_{1}$ & $b_{1}$ & $a_{2}$ & $b_{2}$ \\
\hline Value & $1.1 \times 10^{4}$ & 0.035 & 0.03 & -0.4 & 0.06 & $\begin{array}{c}- \\
0.3\end{array}$ \\
\hline
\end{tabular}

The outdoor temperature change is shown in Fig 2. Referring to the electricity price policy of Tianjin., as shown in Fig 4. Under the premise of ensuring the thermal comfort of users, the optimal scheduling method proposed in this paper is used to optimize the heating scheme of the office building.

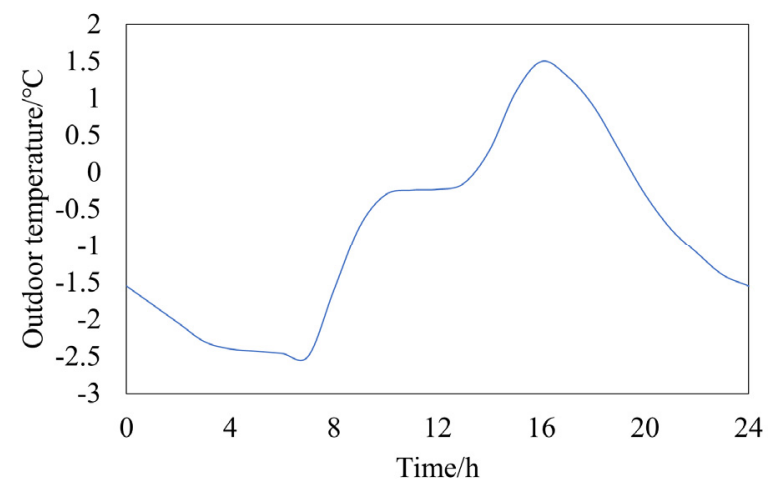

Fig. 2. Outdoor temperature

Working hours of the office building is: 8:30-12:00, 13:00-17:00. Considering the actual arrival and departure of personnel, the indoor temperature setting scheme of the office building is as follows:

7: 00-19:00: the inverter air conditioning system is turned on, and the set temperature is $19^{\circ} \mathrm{C}$ (indoor comfortable temperature range: $18^{\circ} \mathrm{C}-20^{\circ} \mathrm{C}$ ); the air conditioning system is turned off at other times.

\section{2 results analysis}

Fig 3 shows the change of charge and discharge power. Fig 4 shows the electric power of inverter air conditioning before and after optimal scheduling, and
Fig 5 shows indoor temperature before and after optimal scheduling. Power consumption and electricity cost before and after optimal scheduling are shown in Table 2.

By observing Fig 3 and Fig 4, it can be seen that the electricity load can be transferred from peak price period to other price periods to a certain extent after using optimal scheduling scheme which is obtained by the optimal scheduling method proposed in this paper.

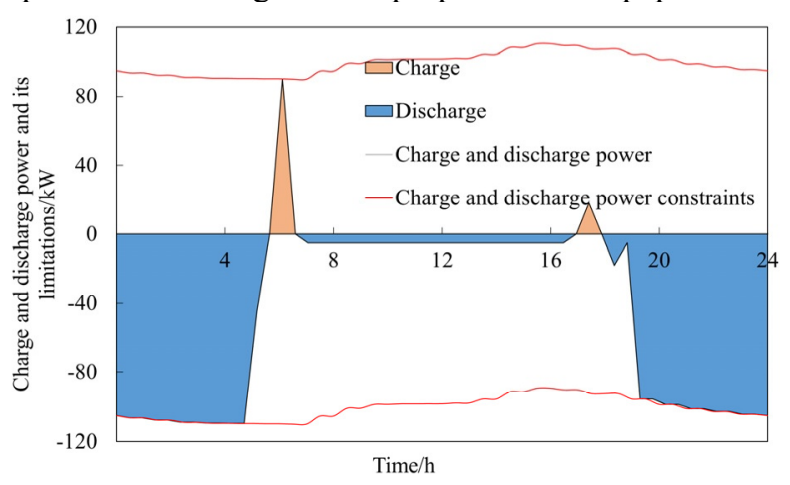

Fig. 3. Change of charge and discharge power

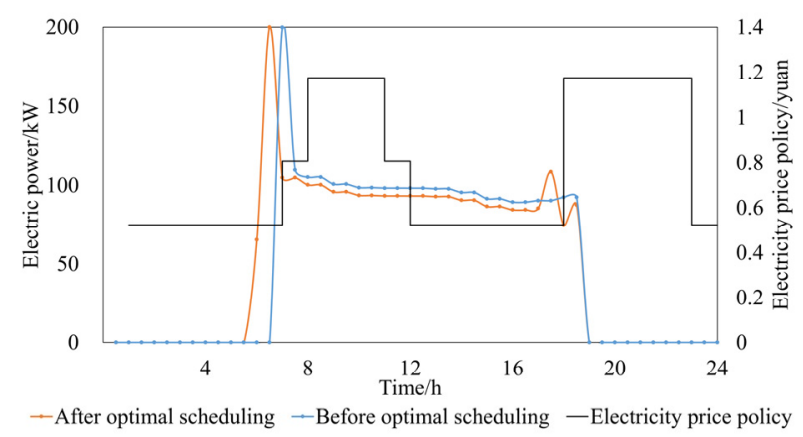

Fig. 4. Electric power of inverter air conditioning before and after optimal scheduling and electricity price policy

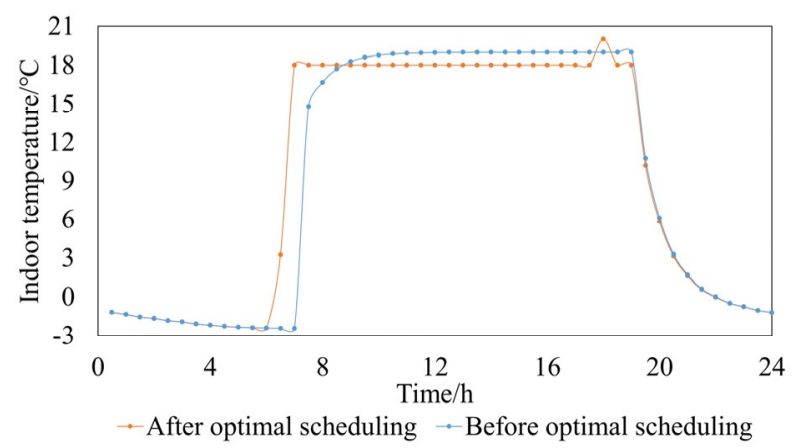

Fig. 5. indoor temperature before and after optimal scheduling

See Table 2 for the power consumption of peak price period before and after the optimal dispatching. After optimal scheduling, the power consumption during peak electricity price period can be reduced by $6.54 \%$, and the electricity cost can be reduced by $1.51 \%$. It should be noted that after optimal scheduling, the power consumption for heating of the whole day has increased. The reason for this phenomenon is: before optimal scheduling, inverter air conditioning turns on for heating at 7:00 according to the room temperature setting scheme. Some moments (such as 7:00) indoor 
temperature does not meet the indoor temperature setting scheme; after optimal scheduling, in order to ensure that the indoor temperature meets the indoor temperature setting scheme, inverter air conditioning starts heating ahead of time, which makes the power consumption for heating increase after optimal scheduling.

Table 2. Power consumption and electricity cost before and after optimal scheduling

\begin{tabular}{|c|c|c|c|}
\hline & $\begin{array}{c}\text { Before } \\
\text { optimal } \\
\text { scheduling }\end{array}$ & $\begin{array}{c}\text { After } \\
\text { optimal } \\
\text { scheduling }\end{array}$ & $\begin{array}{c}\text { Rate of } \\
\text { change }\end{array}$ \\
\hline $\begin{array}{c}\text { Power } \\
\text { consumption } \\
\text { during peak } \\
\text { electricity } \\
\text { price period }\end{array}$ & $396.78 \mathrm{kWh}$ & $370.82 \mathrm{kWh}$ & $-6.54 \%$ \\
\hline $\begin{array}{c}\text { Power } \\
\text { consumption }\end{array}$ & $1212.21 \mathrm{kWh}$ & $1246.55 \mathrm{kWh}$ & $2.83 \%$ \\
\hline $\begin{array}{c}\text { Electricity } \\
\text { cost }\end{array}$ & $964.02 \mathrm{yuan}$ & $949.49 \mathrm{yuan}$ & $-1.51 \%$ \\
\hline
\end{tabular}

in summary, the optimal scheduling method proposed in this paper can reduce the user's electricity cost for heating on the premise of ensuring the user's thermal comfort, respond to the call of power grid to peak cut to a certain extent, and realize the unity of user demand and power grid demand.

\section{Conclusions}

In this paper, the optimal scheduling method based on building virtual energy storage equivalent battery model is proposed. The conclusions are as follows.

1) The building virtual energy storage equivalent battery proposed in this paper exploits the heat storage potential of the building and quantifies it as a battery model, which can ensure the thermal comfort of users, and reduce the user's electricity cost for heating.

2) Building envelope has the ability of heat storage. Considering this factor in the optimal scheduling of building energy consumption can reduce user's electricity cost for heating.

The building virtual energy storage equivalent battery proposed in this paper ignores the influence of the number of furniture in the building, the use of electrical appliances and internal personnel activities. The follow-up research will further consider the influence of these factors and improve the equivalent battery model.

\section{Acknowledgments}

This work was supported by the Science and Technology Project of State Grid Jiangxi Electric Power Company (SGJXDK00DWJS1900282).

\section{References}

1. Hongjie Jia, Yunfei Mu, Xiaodan Yu. Thought about the integrated energy system in China, Electric Power Construction, 36, 16-25 (2015)

2. J. Lizana, R. Chacartegui, A. Barrios-Padura, et al. Advances in thermal energy storage materials and their applications towards zero energy buildings: A critical review, Applied Energy, 203, 219-239 (2017)

3. Research Center for Building Energy Conservation, Tsinghua University. China Annual Development Report on Building Energy Conservation 2020 (Rural Housing Topics), Beijing: China Building Industry Press, (2020)

4. National Energy Administration. Notice on the issuance of the winter clean heating plan in the northern region, http: //www.gov.cn/xinwen/201712/20/content_5248855.html, (2017)

5. Jianxiao Wang, Haiwang Zhong, Zhifang Yang, et al. Exploring the trade-offs between electric heating policy and carbon mitigation in China, Nature communications, 11, (2020)

6. Xiaolong Jin, Yunfei Mu, Hongjie Jia, et al. Model predictive control based multiple-time-scheduling method for microgrid system with smart buildings integrated, Automation of Electric Power Systems, 16, 25-43, (2019)

7. José Sánchez Ramos, Mcarmen Pavón Moreno, Mcarmen Guerrero Delgado, Servando Álvarez Domínguez, Luisa F.Cabeza. Potential of energy flexible buildings:Evaluation of DSM strategies using building thermal mass, Energy \&amp; Buildings, 203, (2019)

8. Tianyang Li, Xingwang Zhao, Wenju Xiao. Regulation technology of air-conditioning load in commercial buildings for balance of power grid peak and valley, Automation of Electric Power Systems, 39, 96-102, (2015)

9. G. Reynders, T. Nuytten, D. Saelens. Potential of structural thermal mass for demand-side management in dwellings, Building and Environment, 64, (2013)

10. Xiaolong Zhang. Model predictive control method for building microgrid considering virtual storage system, Tianjin University, (2018)

11. Reynders G, Diriken J, Saelens D. Generic characterization method for energy flexibility:

Applied to structural thermal storage in residential buildings, Applied Energy, 198, 192-202, (2017)

12. Song, M, Gao, C, Yan, H, \& Yang, J. Thermal battery modeling of inverter air conditioning for demand response, IEEE Transactions on Smart Grid, 9, 5522-5534, (2018)

13. Jonathan Roth, Amory Martin, Clayton Miller, Rishee K. Jain. SynCity: Using open data to create a synthetic city of hourly building energy estimates by integrating data-driven and physics-based methods, Applied Energy, 280, 115981, (2020) 